\title{
BSE-PROPERTY FOR SOME CERTAIN SEGAL AND BANACH ALGEBRAS
}

\author{
MOHAMMAD FOZOUNI ${ }^{1 *}$ and MEHDI NEMATI ${ }^{2}$
}

\begin{abstract}
For a commutative semi-simple Banach algebra $A$ which is an ideal in its second dual we give a necessary and sufficient condition for an essential abstract Segal algebra in $A$ to be a BSE-algebra. We show that a large class of abstract Segal algebras in the Fourier algebra $A(G)$ of a locally compact group $G$ are BSE-algebra if and only if they have bounded weak approximate identities. Also, in the case that $G$ is discrete we show that $A_{\mathrm{cb}}(G)$ is a BSE-algebra if and only if $G$ is weakly amenable. We study the BSE-property of some certain Segal algebras implemented by local functions that were recently introduced by J. Inoue and S.-E. Takahasi. Finally we give a similar construction for the group algebra implemented by a measurable and sub-multiplicative function.
\end{abstract}

\section{Introduction and Preliminaries}

Let $G$ be a locally compact abelian group. A subspace $\mathcal{S}$ of $L^{1}(G)$ is called a (Reiter) Segal algebra if it satisfies the following conditions:

(1) $\mathcal{S}$ is dense in $L^{1}(G)$.

(2) $\mathcal{S}$ is a Banach space under some norm $\|\cdot\|_{\mathcal{S}}$ such that $\|f\|_{1} \leq\|f\|_{\mathcal{S}}$ for each $f \in \mathcal{S}$.

(3) $L_{y} f$ is in $\mathcal{S}$ and $\|f\|_{\mathcal{S}}=\left\|L_{y} f\right\|_{\mathcal{S}}$ for all $f \in \mathcal{S}$ and $y \in G$ where $L_{y} f(x)=$ $f\left(y^{-1} x\right)$.

(4) For all $f \in \mathcal{S}$, the mapping $y \longrightarrow L_{y} f$ is continuous with respect to $\|\cdot\|_{\mathcal{S}}$. In [1], J. T. Burnham with changing $L^{1}(G)$ by an arbitrary Banach algebra $A$, gave a generalization of Segal algebras and introduced the notion of an abstract Segal algebra. Recall that a Banach algebra $B$ is an abstract Segal algebra of a Banach algebra $A$ if

(1) $B$ is a dense left ideal in $A$,

(2) there exists $M>0$ such that $\|b\|_{A} \leq M\|b\|_{B}$ for each $b \in B$,

(3) there exists $C>0$ such that $\|a b\|_{B} \leq C\|a\|_{A}\|b\|_{B}$ for each $a, b \in B$.

Recently, J. Inoue and S.-E. Takahasi in [11] investigated abstract Segal algebras in a non-unital commutative semi-simple regular Banach algebra $A$ such that $A$ has a bounded approximate identity in $A_{c}$ where

$$
A_{c}=\{a \in A: \widehat{a} \text { has compact support }\},
$$

Date: Received: xxxxxx; Revised: yyyyyy; Accepted: zzzzzz.

*Corresponding author.

2010 Mathematics Subject Classification. Primary 46H05; Secondary: 46J10, 22D05, 43A25.

Key words and phrases. Banach algebra, Segal algebra, BSE-algebra, locally compact group, Fourier algebra. 
and $\widehat{a}$ denotes the Gel'fand transform of $a$. It is well-known that $L^{1}(G)$ is a commutative semi-simple regular Banach algebra with a bounded approximate identity with compact support; see [14].

A commutative Banach algebra $A$ is without order if for $a \in A$, the condition $a A=\{0\}$ implies $a=0$ or equivalently $A$ does not have any non-zero annihilator. For example if $A$ has an approximate identity, then it is without order. A linear operator $T$ on $A$ is called a multiplier if it satisfies $a T(b)=T(a) b$ for all $a, b \in A$. Suppose that $\mathcal{M}(A)$ denotes the space of all multipliers of the Banach algebra $A$. If $\Delta(A)$ denotes the space of all characters of $A$; that is, non-zero homomorphisms from $A$ into $\mathbb{C}$, then for each $T \in \mathcal{M}(A)$, there exists a unique bounded continuous function $\widehat{T}$ on $\Delta(A)$ such that $\widehat{T(a)}(\phi)=\widehat{T}(\phi) \widehat{a}(\phi)$ for all $a \in A$ and $\phi \in \Delta(A)$; see [14, Proposition 2.2.16]. Let $\widehat{\mathcal{M}(A)}$ denote the space of all $\widehat{T}$ corresponding to $T \in \mathcal{M}(A)$.

A bounded continuous function $\sigma$ on $\Delta(A)$ is called a BSE-function if there exists a constant $C>0$ such that for each $\phi_{1}, \ldots, \phi_{n} \in \Delta(A)$ and complex numbers $c_{1}, \ldots, c_{n}$, the inequality

$$
\left|\sum_{i=1}^{n} c_{i} \sigma\left(\phi_{i}\right)\right| \leq C\left\|\sum_{i=1}^{n} c_{i} \phi_{i}\right\|_{A^{*}}
$$

holds. Let $C_{\mathrm{BSE}}(\Delta(A))$ be the set of all BSE-functions.

A without order commutative Banach algebra $A$ is called a BSE-algebra if

$$
C_{\mathrm{BSE}}(\Delta(A))=\widehat{\mathcal{M}(A)} \text {. }
$$

The theory of BSE-algebras was first introduced and investigated by Takahasi and Hatori; see [20] and two other notable works [15, 13]. In [13], the authors answered a question raised in [20]. Examples of BSE-algebras are the group algebra $L^{1}(G)$ of a locally compact abelian group $G$, the Fourier algebra $A(G)$ of a locally compact amenable group $G$, all commutative $C^{*}$-algebras, the disk algebra, and the Hardy algebra on the open unit disk.

A net $\left\{a_{\alpha}\right\}$ in $A$ is called a bounded weak approximate identity (BWAI) for $A$ if $\left\{a_{\alpha}\right\}$ is bounded in $A$ and

$$
\lim _{\alpha} \phi\left(a_{\alpha} a\right)=\phi(a) \quad(\phi \in \Delta(A), a \in A)
$$

or equivalently, $\lim _{\alpha} \phi\left(a_{\alpha}\right)=1$ for each $\phi \in \Delta(A)$. Clearly, each BAI of $A$ is a BWAI and the converse is not valid in general; see [12] and [16]. Note that bounded weak approximate identities are important to decide whether a commutative Banach algebra is a BSE-algebra or not. For example, if $A$ is a Banach algebra then $\widehat{\mathcal{M}(A)} \subseteq C_{\mathrm{BSE}}(\Delta(A))$ if and only if $A$ has a BWAI; [20, Corollary 5]. Also, it was shown in [10] that a Segal algebra $S(G)$ on a locally compact abelian group $G$ is a BSE-algebra if and only if $S(G)$ has a BWAI.

For undefined concepts and notations appearing in the sequel, one can consult $[3,14]$.

The outline of the next sections is as follows:

In $\S 2$, for a commutative semi-simple Banach algebra $A$ which is an ideal in its second dual we give a necessary and sufficient condition for an essential abstract 
Segal algebra in $A$ to be a BSE-algebra. We show that a large class of abstract Segal algebras in the Fourier algebra $A(G)$ of a locally compact group $G$ are BSEalgebras if and only if they have bounded weak approximate identities. Also, for discrete groups $G$, we show that $A_{\mathrm{cb}}(G)$ is a BSE-algebra if and only if $G$ is weakly amenable.

In $\S 3$, we study the BSE-property of the Segal algebra $A_{\tau(n)}$ in $A$ which was introduced by Inoue and Takahasi and in the case that $\left(A,\|\cdot\|_{X}\right)$ is a BSEalgebra, we show that $A_{\tau(n)}$ is a BSE-algebra if and only if $\tau$ is bounded where $\tau: X \longrightarrow \mathbb{C}$ is a certain continuous function. Also, we compare the BSE-property between $A$ and $A_{\tau(n)}$. In $\S 4$, motivated by the definition of $A_{\tau(n)}$, for an arbitrary locally compact (abelian) group $G$, and a measurable sub-multiplicative function $\tau: G \longrightarrow \mathbb{C}^{\times}$, we define the Banach algebra $L^{1}(G)_{\tau(n)}$. Then we investigate the BSE-property of this algebra.

\section{BSE-Abstract Segal Algebras}

Suppose that $B$ is an abstract Segal algebra of Banach algebra $A$. Endow $\Delta(A)$ and $\Delta(B)$ with the Gel'fand topology, the map $\left.\varphi \mapsto \varphi\right|_{B}$ is a homeomorphism from $\Delta(A)$ onto $\Delta(B)$; see [1, Theorem 2.1].

Let $B$ be an abstract Segal algebra with respect to $A$. We say that $B$ is essential if $\langle A B\rangle$ is $\|\cdot\|_{B}$-dense in $B$, where $\langle A B\rangle$ denotes the linear span of $A B=\{a b: a \in A, b \in B\}$.

Theorem 2.1. Let $A$ be a semi-simple commutative Banach algebra which is an ideal in its second dual $A^{* *}$. Suppose that $B$ is an essential abstract Segal algebra in $A$. Then the following statements are equivalent.

(i) $B$ is a BSE-algebra.

(ii) $B=A$ and $A$ is a BSE-algebra.

Proof. Suppose that $B$ is a BSE-algebra. Then by [20, Corollary 5], $B$ has a BWAI, say $\left(g_{\gamma}\right)_{\gamma}$. It is clear that $\left(g_{\gamma}\right)_{\gamma}$ is also a BWAI for $A$. So, by [15, Theorem 3.1] $A$ is a BSE-algebra and has a bounded approximate identity, say $\left(e_{\alpha}\right)_{\alpha}$. By the Cohen factorization theorem $A B$ is a closed linear subspace of $B$. But $B$ is essential, so $B=A B$. Now, let $b \in B$. Then $b=c a$ for some $c \in B$ and $a \in A$. Since $A$ is an ideal in its second dual, it follows that the operator $\rho_{a}: A \rightarrow A$ defined by $\rho_{a}\left(a^{\prime}\right)=a a^{\prime},\left(a^{\prime} \in A\right)$ is weakly compact; see [4, Lemma 3]. Letting $\iota: B \hookrightarrow A$ and $L_{c}: A \rightarrow B: a^{\prime} \rightarrow c a^{\prime}, \rho_{b}=L_{c} \circ \rho_{a} \circ \iota$ is weakly compact by [2, Proposition VI.5.2]. Therefore, $B$ is an ideal in its second dual. Since by [1, Theorem 2.1] $B$ is semi-simple, [15, Theorem 3.1] implies that $B$ has a bounded approximate identity. Thus $A=B$ by [1, Theorem 1.2]. That (ii) implies (i) is trivial.

Example 2.2. Let $G$ be a locally compact group and let $A(G)$ be the Fourier algebra of $G$. It was shown in [15, Theorem 5.1] that $A(G)$ is a BSE-algebra if and only if $G$ is amenable. Moreover, $A(G)$ is an ideal in its second dual if and only if $G$ is discrete; see [6, Lemma 3.3]. Therefore, by Theorem 2.1 if $G$ is discrete, then an essential abstract Segal algebra $S A(G)$ in $A(G)$ is a BSE-algebra if and only if $S A(G)=A(G)$ and $G$ is amenable. 
Let $G$ be a locally compact group and let $L^{r}(G)$ be the Lebesgue $L^{r}$-space of $G$, where $1 \leq r<\infty$. Then

$$
S A^{r}(G):=L^{r}(G) \cap A(G)
$$

with the norm \|\|$f\|\|=\|f\|_{r}+\|f\|_{A(G)}$ and the pointwise product is an abstract Segal algebra in $A(G)$.

Corollary 2.3. Let $G$ be a discrete group and let $1 \leq r \leq 2$. Then $S A^{r}(G)$ is a BSE-algebra if and only if $G$ is finite

Proof. If $G$ is finite, then $S A^{r}(G)=A(G)$. So, the result follows from Theorem 2.1.

For the converse, first note that $S A^{r}(G)=l^{r}(G)$ and the norms $\|\cdot\|_{r}$ and \|\|$\cdot \|$ on $S A^{r}(G)$ are equivalent by the open mapping theorem. In fact, $l^{2}(G) \subseteq$ $\delta_{e} * l^{2}(G) \subseteq A(G)$, where $\delta_{e}$ is the point mass at the identity element $e$ of $G$. So, if $1 \leq r \leq 2$, then $l^{r}(G) \subseteq l^{2}(G)$ and

$$
l^{r}(G)=l^{r}(G) \cap l^{2}(G) \subseteq S A^{r}(G) \subseteq l^{r}(G) .
$$

Moreover, it is clear that $l^{r}(G)$ has an approximate identity and consequently it is an essential abstract Segal algebra in $A(G)$. Therefore, if $S A^{r}(G)=l^{r}(G)$ is a BSE-algebra, then $A(G)=l^{r}(G)$ by Example 2.2. Thus, $A(G)=l^{2}(G)$, is a reflexive predual of a $W^{*}$-algebra. This implies, as is known, that $A(G)$ is finite dimensional; see [18]. Thus $G$ is finite, which completes the proof.

For a locally compact group $G$, we recall that $A(G)$ is always an ideal in the Fourier-Stieltjes algebra $B(G)$ and note that $\mathcal{M}(A(G))=B(G)$ when $G$ is amenable. The spectrum of $A(G)$ can be canonically identified with $G$. More precisely, the map $x \longrightarrow \varphi_{x}$ where $\varphi_{x}(u)=u(x)$ for all $u \in A(G)$ is a homeomorphism from $G$ onto $\Delta(A(G))$. Note that by [1, Theorem 2.1], $\Delta(S A(G))=$ $\Delta(A(G))=G$.

Theorem 2.4. Let $G$ be a locally compact group and let $S A(G)$ be an abstract Segal algebra in $A(G)$ such that $B(G) \subseteq \mathcal{M}(S A(G))$, i.e., for each $u \in B(G)$, we have $u S A(G) \subseteq S A(G)$. Then $S A(G)$ is a BSE-algebra if and only if $S A(G)$ has a BWAI.

Proof. By [20, Corollay 5], if $S A(G)$ is a BSE-algebra, then it has a BWAI.

Conversely, suppose that $S A(G)$ has a BWAI, say $\left(e_{\gamma}\right)_{\gamma}$. Then $\widehat{\mathcal{M}(S A(G)} \subseteq \subseteq$ $C_{B S E}(\Delta(S A(G)))$ by [20, Corollary 5]. Moreover, it is clear that $\left(e_{\gamma}\right)_{\gamma}$ is also a BWAI for $A(G)$. Consequently, we conclude that $G$ is amenable by [15, Theorem 5.1]. Now, we need to show the reverse inclusion. Since $S A(G)$ is an abstract Segal algebra in $A(G)$, there exists $M>0$ such that $\|u\|_{A(G)} \leq M\|u\|_{S A(G)}$ for all $u \in S A(G)$. Thus, for any $x_{1}, \ldots, x_{n} \in G$ and $c_{1}, \ldots, c_{n} \in \mathbb{C}$,

$$
\left\|\sum_{j=1}^{n} c_{j} \varphi_{x_{j}}\right\|_{S A(G)^{*}} \leq M\left\|\sum_{j=1}^{n} c_{j} \varphi_{x_{j}}\right\|_{A(G)^{*}} .
$$


This implies that

$$
\begin{aligned}
C_{B S E}(\Delta(S A(G))) & \subseteq C_{B S E}(\Delta(A(G))) \\
& =\widehat{B(G)} \\
& \subseteq \mathcal{M}(\widehat{S A(G)}) .
\end{aligned}
$$

Hence, $S A(G)$ is a BSE-algebra.

Example 2.5. (1) Let $G$ be a locally compact group and let $1 \leq r<\infty$. Now, since $\|u\|_{\infty} \leq\|u\|_{B(G)}$ for all $u \in B(G)$, it follows that $u L^{r}(G) \subseteq L^{r}(G)$. This implies that $B(G) \subseteq \mathcal{M}\left(S A^{r}(G)\right)$. Thus $S A^{r}(G)$ is a BSE-algebra if and only if it has a BWAI .

(2) Let $S_{0}(G)$ be the Feichtinger Segal algebra in $A(G)$. Then $B(G) \subseteq \mathcal{M}\left(S_{0}(G)\right)$; see [19, Corollary 5.2]. Thus $S_{0}(G)$ is a BSE-algebra if and only if it has a BWAI

Corollary 2.6. Let $G$ be a locally compact group and let $S A(G)$ be an essential abstract Segal algebra in $A(G)$. Then $S A(G)$ is a BSE-algebra if and only if $S A(G)$ has a BWAI.

Proof. Suppose that $S A(G)$ has a BWAI. Hence $G$ is amenable, therefore $A(G)$ has a bounded approximate identity. Now, by the same argument as the proof of Theorem 2.1 we can show that $S A(G)=A(G) S A(G)$. Consequently, for each $u \in B(G)$

$$
u S A(G)=u A(G) S A(G) \subseteq A(G) S A(G)=S A(G),
$$

which implies that $B(G) \subseteq \mathcal{M}(S A(G))$. Hence, $S A(G)$ is a BSE-algebra by Theorem 2.4.

Suppose that $G$ is a locally compact group and $\mathcal{M}_{\mathrm{cb}} A(G)$ denotes the Banach algebra of completely bounded multipliers of $A(G)$, that is, the continuous and bounded functions $\nu$ on $G$ such that $\nu A(G) \subseteq A(G)$ and the map $L_{\nu}$ defined by $L_{\nu}(u)=\nu u$ is completely bounded; see [5] for a complete course on operator space theory. Note that, by $A(G)=V N(G)_{*}, \mathcal{M}_{\mathrm{cb}} A(G)$ is a completely contractive Banach algebra, where $V N(G)$ is the group von Neumann algebra. It is wellknown that $A(G) \subseteq B(G) \subseteq \mathcal{M}_{\mathrm{cb}} A(G)$. Now, let

$$
A_{\mathrm{cb}}(G)=\overline{A(G)} \|^{\|\cdot\|_{\mathcal{M}_{\mathrm{cb}} A(G)}} \text {. }
$$

This algebra was first introduced by Forrest in [7]. See also some recent works $[8,9,17]$ for more details and properties.

We end this section with the following result regarding the BSE-property of $A_{\mathrm{cb}}(G)$ in the case that $G$ is discrete. Recall that a locally compact group $G$ is said to be weakly amenable if $A(G)$ has an approximate identity which is bounded in $\|\cdot\|_{A_{\mathrm{cb}}(G)}$ or equivalently $A_{\mathrm{cb}}(G)$ has a bounded approximate identity; see [8, Proposition 1].

Theorem 2.7. Suppose that $G$ is a discrete locally compact group. Then $A_{\mathrm{cb}}(G)$ is a BSE-algebra if and only if $G$ is weakly amenable.

Proof. By [17, Lemma 4.1] we know that $G$ is discrete if and only if $A_{\mathrm{cb}}(G)$ is a closed ideal in its second dual. Also, it is clear that $A_{\mathrm{cb}}(G)$ is commutative and semi-simple. Now the result follows by [15, Theorem 3.1]. 


\section{Segal Algebras Implemented by Local Functions}

In this section we focus on a certain Segal algebra that was recently introduced by Inoue and Takahasi. Let $X$ be a non-empty locally compact Hausdorff space. A subalgebra $A$ of $C_{0}(X)$ is called a Banach function algebra if $A$ separates strongly the points of $X$ (that is, for each $x, y \in X$ with $x \neq y$, there exists $f \in A$ such that $f(x) \neq f(y)$ and for each $x \in X$, there exists $f \in A$ such that $f(x) \neq 0)$ and with a norm $\|\cdot\|,(A,\|\cdot\|)$ is a Banach algebra.

Suppose that $(A,\|\cdot\|)$ is a natural regular Banach function algebra on a locally compact, non-compact Hausdorff space $X$ with a bounded approximate identity $\left\{e_{\alpha}\right\}$ in $A_{c}$. We recalling the following definitions from [11].

Definition 3.1. A complex-valued continuous function $\sigma$ on $X$ is called a local $A$-function if for all $f \in A_{c}, f \sigma \in A$. The set of all local $A$-functions is denoted by $A_{\text {loc }}$

Definition 3.2. For positive integer $n$ and a continuous complex-valued function $\tau$ on $X$, put

$$
\begin{aligned}
A_{\tau(n)} & =\left\{f \in A: f \tau^{k} \in A \quad(0 \leq k \leq n)\right\}, \\
\|f\|_{\tau(n)} & =\sum_{k=0}^{n}\left\|f \tau^{k}\right\| .
\end{aligned}
$$

In the sequel of this section, suppose that $n$ is a constant positive integer and $\tau \in A_{\mathrm{loc}}$.

By [11, Theorem 5.4], if $\tau \in A_{\text {loc }}$, then $\left(A_{\tau(n)},\|\cdot\|_{\tau(n)}\right)$ is a Segal algebra in $A$ such that $\Delta\left(A_{\tau(n)}\right)=\Delta(A)=X$, that is, $x \longrightarrow \phi_{x}$ is a homeomorphism from $X$ onto $\Delta\left(A_{\tau(n)}\right)$.

Also, one can see that $A_{\tau(n)}$ is a Banach function algebra, because for each $x, y \in X$ with $x \neq y$, there exists $f \in A_{\tau(n)}$ such that $f(x)=\phi_{x}(f) \neq \phi_{y}(f)=$ $f(y)$ and by using the Urysohn lemma for each $x \in X$, there exists $f \in A_{\tau(n)}$ with $f(x) \neq 0$. Note that by [11, Theorem 3.5], $A_{\tau(n)}$ is Tauberian. Recall that a Banach algebra $A$ is Tauberian if $A_{c}$ is dense in $A$.

The following theorem is one of our main results in this section.

Theorem 3.3. Suppose that $\left(A,\|\cdot\|_{X}\right)$ is a BSE-algebra where $\|\cdot\|_{X}$ is the uniform norm. Then the following statements are equivalent.

(i) $A_{\tau(n)}$ is a BSE-algebra.

(ii) $\tau$ is bounded.

Proof. (i) $\rightarrow$ (ii). Suppose that $A_{\tau(n)}$ is a BSE-algebra. Then it has a BWAI, say $\left\{f_{\alpha}\right\}$. So, there exists a constant $M>0$ such that

$$
\left\|f_{\alpha}\right\|_{\tau(n)}<M, \quad \lim _{\alpha} f_{\alpha}(x)=1 \quad(x \in X) .
$$

On the other hand, $\left\|f_{\alpha} \tau\right\|_{X} \leq\left\|f_{\alpha}\right\|_{\tau(n)}$, hence we have

$$
|\tau(x)| \leq M \quad(x \in X) .
$$

Therefore, $\tau$ is bounded. 
(ii) $\rightarrow$ (i). Let $\tau$ be bounded by $M$, that is, $\|\tau\|_{X}<M$. Clearly, $A_{c} \subseteq A_{\tau(n)}$. For each $f \in A$, there exists a net $\left\{f_{\alpha}\right\}$ in $A_{c}$ such that $\left\|f_{\alpha}-f\right\|_{X} \longrightarrow 0$. Now, $\left\{f_{\alpha} \tau\right\}$ is in $A$ and $f \tau=\lim _{\alpha} f_{\alpha} \tau$, since

$$
\left\|f \tau-f_{\alpha} \tau\right\|_{X}=\left\|\left(f-f_{\alpha}\right) \tau\right\|_{X} \leq M\left\|f-f_{\alpha}\right\|_{X} \longrightarrow 0 .
$$

So, $f \tau \in A$. Similarly, one can see that $f \tau^{k}$ for each $1<k \leq n$ is in $A$. Therefore, $f \in A_{\tau(n)}$. Hence $A=A_{\tau(n)}$. Finally, for each $f \in A$

$$
\|f\|_{X} \leq\|f\|_{\tau(n)} \leq\|f\|_{X}\left(\sum_{k=0}^{n} M^{k}\right) .
$$

Therefore, $A_{\tau(n)}$ is topologically isomorphic to $A$, so $A_{\tau(n)}$ is a BSE-algebra.

Recall that $\tau \in A_{\text {loc }}$ is called a rank $\infty$ local $A$-function, if for each $k=$ $0,1,2, \cdots$, the inclusion $A_{\tau(k)} \supsetneqq A_{\tau(k+1)}$ holds. By [11, Proposition 8.2 (ii)], if $\|\tau\|_{X}=\infty$, then $\tau$ is a rank $\infty$ local $A$-function.

As an application of the above theorem, we give the following result which provide for us examples of Banach algebras without any BWAI.

Corollary 3.4. Let $A=C_{0}(\mathbb{R})$ and $\tau(x)=x$ for every $x \in \mathbb{R}$. Then

$$
A \supsetneqq A_{\tau(1)} \supsetneqq A_{\tau(2)} \supsetneqq \cdots \supsetneqq A_{\tau(n)} \supsetneqq \cdots .
$$

For each $k=1,2,3, \cdots, A_{\tau(k)}$ is not a BSE-algebra and has no BWAI.

Proof. By [20, Theorem 3], if $A=C_{0}(\mathbb{R})$, we know that $A$ is a BSE-algebra. Since for each $x \in \mathbb{R}$, there exists $f \in A$ such that $f=\tau$ on a neighborhood of $x$, hence by [11, Proposition 7.2], $\tau$ is an element of $A_{\text {loc }}$. But $\tau$ is not bounded and this implies that $A_{\tau(k)}$ is not a BSE-algebra by Theorem 3.3. Also, since $\tau$ is not bounded, $\tau$ is a rank $\infty$ local $A$-function, hence we have equation 3.1. Finally, if $A_{\tau(k)}$ has a BWAI, then similar to the proof of Theorem 3.3 one has $\|\tau\|_{\mathbb{R}}<\infty$ which is impossible.

By the above corollary if $A$ is a BSE-algebra, then $A_{\tau(n)}$ is not necessarily a BSE-algebra. For the converse we have the following proposition.

Proposition 3.5. Suppose that $\tau \in A_{\mathrm{loc}}$ and $A$ is an ideal in its second dual. If $A_{\tau(n)}$ is a BSE-algebra, then $A$ is a BSE-algebra.

Proof. If $\left\{e_{\alpha}\right\}$ is a b.a.i. for $A$, then it follows from our definition of $A_{\tau(n)}$ that $\left\{e_{\alpha}\right\}$ is also an approximate identity for $A_{\tau(n)}$ when viewed as a Banach $A$-module.

So, $A_{\tau(n)}$ is an essential abstract Segal algebra with respect to $A$, because for each $b \in A_{\tau(n)},\left\|b-e_{\alpha} b\right\|_{\tau(n)} \rightarrow 0$. Now, using Theorem 2.1 we have the result.

We do not know whether Proposition 3.5 fails if the assumption that $A$ is an ideal in its second dual is dropped.

Remark 3.6. If $X$ is a discrete space, then $A$ is an ideal in its second dual. Since $A$ is a semi-simple commutative and Tauberian Banach algebra, using [15, Remark 3.5], we conclude that $A$ is an ideal in its second dual. 


\section{A Construction on Group Algebras}

Let $G$ be a locally compact group and let $L^{1}(G)$ be the space of all measurable and integrable complex-valued functions (equivalent classes with respect to the almost everywhere equality relation) on $G$ with respect to the left Haar measure of $G$. The convolution product of the functions $f$ and $g$ in $L^{1}(G)$ is defined by

$$
f * g(x)=\int_{G} f(y) g\left(y^{-1} x\right) d y
$$

For each $f \in L^{1}(G)$, let $\|f\|_{1}=\int_{G}|f(x)| d x$. It is well-known that $L^{1}(G)$ endowed with the norm $\|\cdot\|_{1}$ and the convolution product is a Banach algebra called the group algebra of $G$; see [3, Section 3.3] for more details.

The following lemma was proved by Bochner and Schoenberg for $G=\mathbb{R}$ in (1934) and by Eberlein for general locally compact abelian (LCA) groups in (1955). Here, we explicitly observe that a result due to E. Kaniuth and A. Ülger gives another proof.

Recall that for a LCA group $G$, the dual group of $G, \widehat{G}$ is defined as the set of all continuous homomorphisms from $G$ to $\mathbb{T}$ where $\mathbb{T}=\{z \in \mathbb{C}:|z|=1\}$. It is well-known that $\widehat{G}$ is a LCA group with the pointwise-defined operation.

Lemma 4.1. Suppose that $G$ is LCA group. Then $L^{1}(G)$ is a BSE-algebra.

Proof. We know that $L^{1}(G)$ is isometrically isomorphic to $A(\widehat{G})$. But $\widehat{G}$ is amenable. Therefore, by $\left[15\right.$, Theorem 5.1] $A(\widehat{G})$, and so $L^{1}(G)$, is a BSEalgebra.

In the sequel, motivated by the construction of $A_{\tau(n)}$ in the preceding section, we introduce a subalgebra of the group algebra $A=L^{1}(G)$ where $G$ is a locally compact group.

Recall that $\varphi: G \longrightarrow \mathbb{C}^{\times}$is sub-multiplicative if

$$
|\varphi(x y)| \leq|\varphi(x) \| \varphi(y)| \quad(x, y \in G)
$$

where $\mathbb{C}^{\times}$denotes the multiplicative group of non-zero complex numbers.

For a measurable sub-multiplicative function $\tau: G \longrightarrow \mathbb{C}^{\times}$and each $n \in \mathbb{N}$, put

$$
\begin{aligned}
L^{1}(G)_{\tau(n)} & =\left\{f \in L^{1}(G): f \tau, \ldots, f \tau^{n} \in L^{1}(G)\right\} \\
\|f\|_{\tau(n)} & =\sum_{k=0}^{n}\left\|f \tau^{k}\right\|_{1} \quad\left(f \in L^{1}(G)_{\tau(n)}\right) .
\end{aligned}
$$

As the first result in this section, we show that $L^{1}(G)_{\tau(n)}$ is a Banach algebra as follows.

Proposition 4.2. $L^{1}(G)_{\tau(n)}$ is a Banach algebra with the convolution product and the norm $\|\cdot\|_{\tau(n)}$. 
Proof. For each $f, g \in L^{1}(G)_{\tau(n)}$ and $1 \leq k \leq n$, we have

$$
\begin{aligned}
\left\|(f * g) \tau^{k}\right\|_{1} & \leq \iint\left|f(y)\left\|g\left(y^{-1} x\right)\right\| \tau^{k}(x)\right| d y d x \\
& =\iint\left|f(y)\left\|g\left(y^{-1} x\right)\right\| \tau^{k}(x)\right| d x d y \\
& =\iint\left|f(y)\|g(x)\| \tau^{k}(y x)\right| d x d y \\
& \leq\left\|f \tau^{k}\right\|_{1}\left\|g \tau^{k}\right\|_{1} .
\end{aligned}
$$

For each $1 \leq k \leq n$, since $\tau$ is measurable, $(f * g) \tau^{k}$ is measurable and by the above inequality $\left\|(f * g) \tau^{k}\right\|_{1}<\infty$. Therefore, $f * g$ is in $L^{1}(G)_{\tau(n)}$. Also, we have

$$
\begin{aligned}
\|f * g\|_{\tau(n)} & =\|f * g\|_{1}+\sum_{k=1}^{n}\left\|(f * g) \tau^{k}\right\|_{1} \\
& \leq\|f\|_{1}\|g\|_{1}+\sum_{k=1}^{n}\left\|f \tau^{k}\right\|_{1}\left\|g \tau^{k}\right\|_{1} \\
& \leq\|f\|_{\tau(n)}\|g\|_{\tau(n)} .
\end{aligned}
$$

To see the completeness of $\|\cdot\|_{\tau(n)}$, let $\left\{f_{i}\right\}$ be a Cauchy sequence in $L^{1}(G)_{\tau(n)}$. So, there exist $f \in L^{1}(G)$ and $g_{k} \in L^{1}(G)$ for each $1 \leq k \leq n$ such that

$$
\lim _{i \rightarrow \infty}\left\|f_{i}-f\right\|_{1}=0, \quad \lim _{i \rightarrow \infty}\left\|f_{i} \tau^{k}-g_{k}\right\|_{1}=0 .
$$

Since $\lim _{i \rightarrow \infty}\left\|f_{i}-f\right\|_{1}=0$, there exists a subsequence $\left\{f_{i_{m}}\right\}$ such that

$$
\lim _{i_{m}} f_{i_{m}}(x)=f(x) \text { a.e., for } x \in G \text {. }
$$

Also, since $\lim _{i_{m}}\left\|f_{i_{m}} \tau^{k}-g_{k}\right\|_{1}=0$, there exists a subsequence $\left\{f_{i_{m, k}}\right\}$ of $\left\{f_{i_{m}}\right\}$ such that

$$
\lim _{i_{m, k}} f_{i_{m, k}}(x) \tau^{k}(x)=g_{k}(x) \text { a.e., for } x \in G .
$$

Therefore, $f \tau^{k}=g_{k}$ a.e., so, $f$ is an element of $L^{1}(G)_{\tau(n)}$ such that

$$
\begin{aligned}
\left\|f_{i}-f\right\|_{\tau(n)} & =\left\|f_{i}-f\right\|_{1}+\left\|f_{i} \tau-f \tau\right\|_{1}+\cdots+\left\|f_{i} \tau^{n}-f \tau^{n}\right\|_{1} \\
& =\left\|f_{i}-f\right\|_{1}+\left\|f_{i} \tau-g_{1}\right\|_{1}+\cdots+\left\|f_{i} \tau^{n}-g_{n}\right\|_{1} \longrightarrow 0 .
\end{aligned}
$$

Hence, $\left(L^{1}(G)_{\tau(n)},\|\cdot\|_{\tau(n)}\right)$ is complete.

In the sequel, we suppose that $G$ is a LCA group.

Theorem 4.3. If $\tau$ is bounded, then $L^{1}(G)_{\tau(n)}$ is a BSE-algebra.

Proof. If $\tau$ is bounded by $M$, then for each $f \in L^{1}(G)$ and $1 \leq k \leq n$ we have $f \tau^{k} \in L^{1}(G)$ and

$$
\|f\|_{1} \leq\|f\|_{\tau(n)}=\sum_{k=0}^{n}\left\|f \tau^{k}\right\|_{1} \leq\|f\|_{1}\left(\sum_{k=0}^{n} M^{k}\right) .
$$


So, $L^{1}(G)_{\tau(n)}$ is topologically isomorphic to $L^{1}(G)$ and hence it is a BSE-algebra by Lemma 4.1 and [15, Corollary 1.3].

When $\tau$ satisfying $|\tau(x)| \geq 1$ a.e., for $x \in G$, we show in the sequel that $L^{1}(G)_{\tau(n)}$ is in fact a Beurling algebra, the definition of which we now recall.

A weight on $G$ is a measurable function $w: G \longrightarrow(0, \infty)$ such that $w(x y) \leq$ $w(x) w(y)$ for all $x, y \in G$. The Beurling algebra $L^{1}(G, w)$ is defined to be the space of all measurable complex-valued functions $f$ on $G$ such that $\|f\|_{1, w}=$ $\int|f(x)| w(x) d x<\infty$. The Beurling algebra with the convolution product and the norm $\|\cdot\|_{1, w}$ is a Banach algebra with $\Delta\left(L^{1}(G, w)\right)=\widehat{G}(w)$, where $\widehat{G}(w)$ is the space of all non-zero complex-valued continuous homomorphisms $\varphi$ on $G$ such that $|\varphi(x)| \leq w(x)$ for each $x \in G$; see [14].

The space $M(G, w)$ of all complex regular Borel measures $\mu$ on $G$ such that $\mu w \in M(G)$ with convolution product and norm

$$
\|\mu\|_{M(G), w}=\|\mu w\|_{M(G)}=\int w(x) d|\mu|(x)
$$

is a Banach algebra called the weighted measure algebra, where $\mu w$ is defined by

$$
\mu w(B)=\int_{B} w(x) d \mu(x) \text { for each Borel subset } B \text { of } G \text {. }
$$

Proposition 4.4. If $|\tau| \geq 1$ a.e., then $L^{1}(G)_{\tau(n)}$ and $L^{1}\left(G,\left|\tau^{n}\right|\right)$ are topologically isomorphic.

Proof. Suppose that for almost every $x \in G,|\tau(x)| \geq 1$. Clearly if $f \in L^{1}(G)_{\tau(n)}$, then we have $f \in L^{1}\left(G,\left|\tau^{n}\right|\right)$. On the other hand, if $f \in L^{1}\left(G,\left|\tau^{n}\right|\right)$ by applying $|\tau| \geq 1$ a.e., we conclude that $f \in L^{1}(G)_{\tau(n)}$, since for all $0 \leq k \leq n$, $\left|f(x)\left\|\tau^{k}(x)|\leq| f(x)\right\| \tau^{n}(x)\right|$ a.e.. Therefore, $L^{1}\left(G,\left|\tau^{n}\right|\right)=L^{1}(G)_{\tau(n)}$ as two sets. Also, using $|\tau| \geq 1$ a.e., we have

$$
\|f\|_{1, w} \leq\|f\|_{\tau(n)} \leq(n+1)\|f\|_{1, w},
$$

where $w=\left|\tau^{n}\right|$. So, two the norms $\|\cdot\|_{1, w}$ and $\|\cdot\|_{\tau(n)}$ are equivalent, which completes the proof.

Remark 4.5. Note that $L^{1}(G)_{\tau(n)}$ and $L^{1}\left(G,\left|\tau^{n}\right|\right)$ are not equal in general. For example, let $G=R^{+}$be the multiplicative group of all positive real numbers, $n=$ 2 and $\tau(x)=\frac{1}{x}$ for all $x \in R^{+}$. Clearly, $\tau$ is measurable and sub-multiplicative. Also, it is easily verified that $L^{1}(G)_{\tau(2)} \subseteq L^{1}\left(G,\left|\tau^{2}\right|\right)$. Now, take $0<\alpha<1$ and put

$$
f(x)= \begin{cases}0, & 0<x<1 \\ x^{\alpha}, & 1 \leq x\end{cases}
$$

One can easily check that $f$ is in $L^{1}\left(G,\left|\tau^{2}\right|\right)$ but it is not in $L^{1}(G)_{\tau(2)}$. So,

$$
L^{1}\left(G,\left|\tau^{2}\right|\right) \neq L^{1}(G)_{\tau(2)} .
$$

Also, if we put $g(x)=\chi_{(0,1]}$, then $g \in L^{1}(G)$ but $g \notin L^{1}(G)_{\tau(2)}$. Hence,

$$
L^{1}(G) \neq L^{1}(G)_{\tau(2)} \text {. }
$$


Remark 4.6. Although in general $L^{1}\left(G,\left|\tau^{k}\right|\right) \neq L^{1}(G)_{\tau(n)}$ for every integer $k$ with $0 \leq k \leq n$, we do have

$$
\overline{L^{1}(G)_{\tau(n)}}\|\cdot\|_{1,\left|\tau^{k}\right|}=L^{1}\left(G,\left|\tau^{k}\right|\right), \quad(0 \leq k \leq n) .
$$

To see that this holds, observe that $C_{c}(G)$ is dense in $L^{1}\left(G,\left|\tau^{k}\right|\right)$ and similar to $[14$, Lemma $1.3 .5(\mathrm{i})]$, one can see that $C_{c}(G) \subseteq L^{1}(G)_{\tau(n)}$.

Remark 4.7. Let $K \subseteq G$ be a relatively compact neighborhood of $e$. Put

$$
\mathcal{U}_{K}=\{U \subseteq K: U \text { is a relatively compact neighborhood of } e\} .
$$

For each $U \in \mathcal{U}_{K}$, let $f_{U}=\frac{\chi_{U}}{|U|}$, where $|U|$ denotes the Haar measure of $U$. Since $K$ is relatively compact by [14, Lemma 1.3.3], there exists a positive real number $b$ such that $|\tau(x)| \leq b$ for all $x \in K$. So

$$
\left\|f_{U}\right\|_{\tau(n)} \leq 1+b+\ldots+b^{n} \quad\left(U \in \mathcal{U}_{K}\right) \text {. }
$$

Also, similar to the group algebra case, for each $f \in L^{1}(G)_{\tau(n)}$ we have $\| f_{U} * f-$ $f \|_{\tau(n)} \longrightarrow 0$ when $U$ tends to $\{e\}$. Therefore, $\left\{f_{U}\right\}_{U \in \mathcal{U}_{K}}$ is a BAI for $L^{1}(G)_{\tau(n)}$.

Remark 4.8. Using the above remarks one can see that in general $L^{1}(G)_{\tau(n)}$ is not an abstract Segal algebra with respect to $L^{1}(G)$, because $L^{1}(G)_{\tau(n)}$ has a b.a.i and in general $L^{1}(G) \neq L^{1}(G)_{\tau(n)}$. But it is well-known that if $\mathcal{S}$ is an abstract Segal algebra with respect to $L^{1}(G)$ such that $\mathcal{S}$ has a BAI, then $\mathcal{S}=L^{1}(G)$.

Suppose that $G$ is compact and $w$ is a weight on $G$. So, by Lemma 1.3 .3 and Corollary 1.3.4 of [14], there exists positive real number $b$ such that $1 \leq w(x) \leq b$ for all $x \in G$. Hence, using Proposition 4.4 and the proof of Theorem 4.3, we

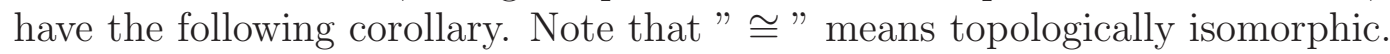

Corollary 4.9. If $G$ is a compact group, then $L^{1}(G)_{\tau(n)}$ is a BSE-algebra and we have the following relations:

$$
L^{1}\left(G,\left|\tau^{n}\right|\right) \cong L^{1}(G)_{\tau(n)} \cong L^{1}(G) .
$$

Remark 4.10. For every integer $k$ with $0 \leq k \leq n$, we have,

$$
L^{1}(G)_{\tau(n)} \subseteq L^{1}\left(G,\left|\tau^{k}\right|\right) .
$$

Therefore,

$$
\widehat{G} \cup \widehat{G}(|\tau|) \cup \ldots \cup \widehat{G}\left(\left|\tau^{n}\right|\right) \subseteq \Delta\left(L^{1}(G)_{\tau(n)}\right) .
$$

Remark 4.11. Put $M(G)_{\tau(n)}=\bigcap_{k=1}^{n} M\left(G,\left|\tau^{k}\right|\right)$ and define the following norm:

$$
\|\mu\|_{\tau(n)}=\sum_{k=0}^{n}\left\|\mu\left|\tau^{k}\right|\right\|_{M(G)} \quad\left(\mu \in M(G)_{\tau(n)}\right) .
$$

A direct use of the convolution product shows that $\left(M(G)_{\tau(n)},\|\cdot\|_{\tau(n)}\right)$ is a normed algebra such that

$$
M(G)_{\tau(n)} \subseteq \mathcal{M}\left(L^{1}(G)_{\tau(n)}\right)
$$

We do not know whether the converse of the above inequality holds or not. Clearly, if $\tau$ is bounded, then $M(G) \cong M(G)_{\tau(n)}$ and so the converse of the above inequality holds by Wendel's Theorem. 
We end this paper with the following questions and conjecture.

Conjecture: We conjecture that Theorem 2.7 is valid for every locally compact group.

Question: Are the converse of Theorem 4.3 and Relation 4.1 hold?

Acknowledgments. The authors would like to thank the referee for his/her suggestions and comments which improve the presentation of the paper especially, giving a shorter proof for Theorem 2.1. The first named author of the paper supported partially by a grant from Gonbad Kavous University.

\section{REFERENCES}

1. J. T. Burnham, Closed ideals in subalgebras of Banach algebras I, Proc. Amer. Math. Soc. 32 (1972), 551-555.

2. J. B. Conway, A course in functional analysis, second ed., Springer-Verlag New York, 1990.

3. H. G. Dales, Banach algebras and automatic continuity, Clarendon press, Oxford, 2000.

4. J. Duncan and S. A. R. Hosseiniun, The second dual of a Banach algebra, Proc. Roy. Soc. Edinburgh Sect. 84 (1979), 309-325.

5. E. G. Effros and Z-J. Ruan, Operator spaces, Clarendon Press, Oxford, 2000.

6. B. Forrest, Arens regularity and discrete groups, Pacific. J. Math. 151 (1991), no. 2, 217227.

7. 114 (1992), no. 4, 965-970.

8. B. E. Forrest, Completely bounded multipliers and ideals in $A(G)$ vanishing on closed subgroups, Contemp. Math. 363 (2004), 89-94.

9. B. E. Forrest, V. Runde, and N. Spronk, Operator amenability of the Fourier algebra in the cb-multiplier norm, Can. J. Math. 59 (2007), 966-980.

10. J. Inoue and S.-E. Takahasi, Constructions of bounded weak approximate identities for Segal algebras on LCA groups, Acta Sci. Math.(Szeged) 66 (2000), 257-271.

11. _ Segal algebras in commutative Banach algebras, Rocky Mountain J. Math. 44 (2014), no. 2, 539-589.

12. C. A. Jones and C. D. Lahr, Weak and norm approximate identities are different, Pacific J. Math. 72 (1977), no. 1, 99-104.

13. Z. Kamali and M. L. Bami, The Bochner-Schoenberg-Eberlein property for $L^{1}\left(\mathbb{R}^{+}\right)$, J. Fourier Anal. Appl. 20 (2014), no. 2, 225-233.

14. E. Kaniuth, A course in commutative Banach algebras, Springer Verlag, Graduate texts in mathematics, 2009.

15. E. Kaniuth and A. Ülger, The Bochner-Schoenberg-Eberlein property for commutative Banach algebras, especially Fourier and Fourier Stieltjes algebras, Trans. Amer. Math. Soc. 362 (2010), 4331-4356.

16. J. Laali and M. Fozouni, On $\Delta$-weak $\phi$-amenable Banach algebras, U. P. B. Sci. Bull. Series A 77 (2015), no. 4, 165-176.

17. M. Nemati, On generalized notions of amenability and operator homology of the Fourier algebra, Quart. J. Math. 68 (2017), no. 3, 781-789.

18. S. Sakai, Weakly compact operators on operators algebra, Pacific. J. Math. 14 (1964), no. 2, 659-664.

19. E. Samei, N. Spronk, and R. Stokke, Biflatness and pseudo-amenability of Segal algebras, Canad. J. Math. 62 (2010), 845-869.

20. S.-E. Takahasi and O. Hatori, Commutative Banach algebras which satisfy a BochnerSchoenberg-Eberlein-type theorem, Proc. Amer. Math. Soc. 110 (1990), 149-158. 
${ }^{1}$ Department of Mathematics, Gonbad Kavous University, P.O.Box 163, GonbadE Kavous, Iran.

E-mail address: fozouni@gonbad.ac.ir

${ }^{2}$ Department of Mathematical Sciences, Isfahan University of Technology, ISFAHAN 84156-83111, IRAN;

E-mail address: m.nemati@cc.iut.ac.ir 